\title{
The Concept of Bloom Taxonomic Revision and Critical Thinking in Fashion Design Learning
}

\author{
Nining Tristantie ${ }^{1}$, Syahril $^{2}$, Armaini Rambe ${ }^{3}$, Juliarti ${ }^{4}$ \\ \{n1n4tristan@gmail.com¹, syahril@gmail.com², ijuliarti@gmail.com ${ }^{3}$, armaini@gmail.com ${ }^{4}$ \} \\ Universitas Negeri Medan, Indonesia ${ }^{1,2,3,4}$ \\ n1n4tristan@gmail.com
}

\begin{abstract}
Bloom Taxonomy is manipulated as a practical way to perform the learning and evaluating. The existing tiers of the revised Bloom Taxonomy by Krathwol are a changing of noun to a verb form. The tiers are applied into the fashion design test questions to gage the frame of thinking in the low and high category (high order thinking skill) in fashion design learning. Based on this category we may find out the tier of students thinking skill to analyze and to interpret the fashion design learning. Questions are compiled using the category emphasizes on high thinking at $\mathrm{C} 4, \mathrm{C} 5$ and $\mathrm{C} 6$. This effort is employed to get the learning goals in order to balance the aspect of cognitive and psychomotor.
\end{abstract}

Keyword: Taxonomic revision, critical thinking, fashion design learning

\section{Introduction}

Education is expected to be anticipative and adaptive to every global changing. It is an absolute as education and industry are two mutual influence relations. It is said so as industry needs human resources who are ready to work, whereas education is charged to be adaptive to response the industry demands which are implemented in learning.

Clothing Education Department in the description of one of its vision as a university that prepares its graduates to be professionals. It is related to its role in the creative industry that is currently developed by strengthening the terms emphasizing on creative skills and innovative activities. The term Creative industry has been used throughout the world to enhance the creativity and innovation activities of its people to support the economic sector [1]. Therefore, the Indonesian creative agency through the ministry of trade revitalizes creative activities and seriously provides full support for the creation of skill-based creative product. The concept of performance usually refers to three pillars namely economic, environmental and social [1]. Creative industries are industries that come from individual creativity, skills, and talents that are potentially create wealth and employment through the exploitation and generation of individual intellectual property and creativity.

Creative people by Kanematsu and Berry are described as people who pursue their goals with intensity, exploratory, optimistic and tolerant in uncertainty. Creative people become reactive to stimuli, are elaborate in the amount of response, flexibility. [2].

The implementation of creativity in pursuing the teaching and learning process in class begins with the preparation of lesson plan. Lectures compile lessons plans based on KKNI that emphasizes independent learning and critical thinking. The final goal is to achieve the 
competencies that have been established in learning fashion design. This Lesson Plan is equipped with an assessment that serves to determine students learning improvement by using evaluation test that can measure the mastery of the given material.

The use of these tests is determined based on critical success indicators in the learning process. In fashion learning, Examining the student's creativity in solving the problem, lectures usually focus on operational verb word using, it doesn't examine the creativity effectively which is oriented to critical thinking. As it is seen from revised taxonomy edition, there are real divisions of high thinking skills and low thinking skills.

Bloom Taxonomy is a ranged classification one of which is used for instructional purpose. Bloom Taxonomy has been revised especially the cognitive realm. David Krathwol - a co writer of original Bloom Taxonomy. According to Anderson et al, Revision is used to improve the tiered structure of thinking, the application can be used in more common language and can articulate more examples.[3]

Taxonomy involves some basic dimensions. Firstly, it is a knowledge domain that involves four knowledge; factual, conceptual, procedural and metacognitive. Second, Factual Knowledge involves basic elements that students should familiarize with discipline and problem solving. Third, Procedural knowledge deals with how to perform something, method of investigation, criteria for doing skills, techniques and methods. Fourth, Metacognitive knowledge involves 'knowledge about cognition in general as well as awareness and knowledge about cognition itself'.'

The domain of knowledge includes the ability to restate the concepts or principles that have been learned, relating to thinking skill, competence in gaining knowledge, recognition, understanding, conceptualization, determination and reasoning. Learning objectives in the cognitive (intellectual) domain or according to Bloom are all activities involving the brain divided into 6 levels according to the lowest to highest levels denoted by $\mathrm{C}$, namely Cognitive (In a book titled Taxonomy of Educational Objectives: Cognitive Domain published by McKey New York, Benjamin Bloom in 1956) known as the Six levels. This is what is often used in formulating learning objectives as $\mathrm{C} 1$ through C6. Revised Taxonomy uses the dimensions of cognitive processes that are interrelated.

\section{Method}

The levels contained in the Bloom's taxonomy revised have been used a basis for educational purposes, test preparation, and curriculum [4] Therefore, fashion design learning uses this taxonomic revision aims to help lectures to understand, organize and implement learning design goals. The concept of fashion learning using the revised Bloom taxonomy is focused on active, cognitive and constructive processes to create meaningful learning. The constructivists understanding, knowledge is the construction (forming) by related something to others (schemata). Knowledge cannot be transferred from the teacher to others, because everyone has their own scheme of what they know. The formation of knowledge is a cognitive process in which the process of assimilation and accommodation occurs to achieve balancing into a new scheme (plural: schemata) where scheme is formed. [5].

Fashion design Learning has stages that are passed by researching methods starting with questions, identification, analyzing, evaluating and creative product creation. These creative processes begin with creative people who produce creative product including every thoughts and actions taken to deliver an original product. 
The popular model suggested by Graham Wallas in 2007. He believes that creative thinking implicates four phases: problem defined preparation), incubation (set the problem aside in long period of time), illumination (when new ideas come) and verification (new ideas investigation). Incubation and illumination phase which show creative thinking is the unconscious mental process.

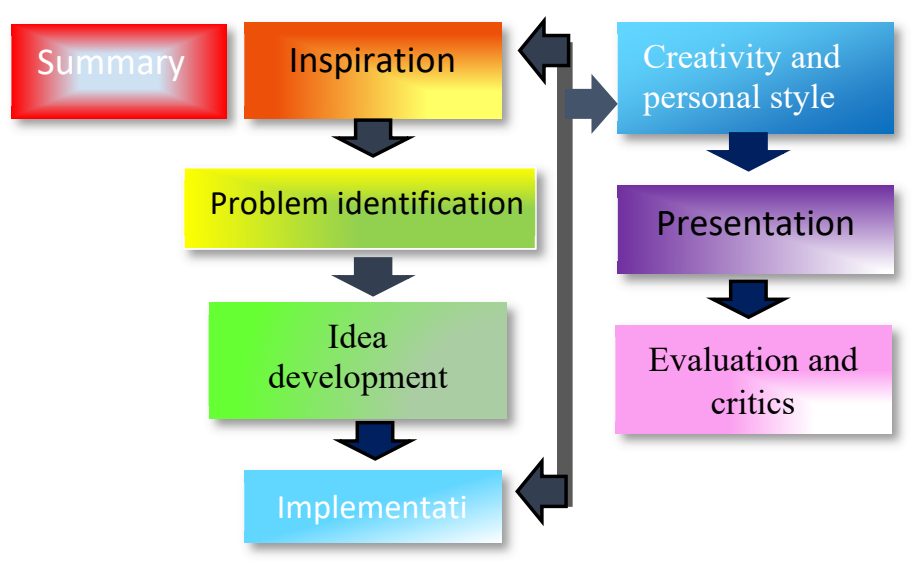

Fig. 1. Creative process of fashion design start from Idea to assessment [6]

In this case, Students are sued to be active in the learning process. In fashion design learning, students are free to express ideas, include selecting problem raised as a topic to be followed up. [7] Students are hoped to be able to construct the significance based on the accepted information. The impact obtained, such as students' complicity in solving problem independently is clearly seen through these learning activities. Then, it will change the students' perspective in solving the problem namely the change from passive to a cognitive and constructive view.

The focus of this learning is to emphasis on what they know in the form of knowledge and how students think (cognitive process) about what they gain during the learning process. Cognitive process dimension contains six categories; memorizing, understanding, applying, analyzing, evaluating and creating. Knowledge dimension contains four categories. They are factual, conceptual, procedural and metacognitive. The existence of these series processes in fashion design need a series of assessments to measure students' critical thinking by applying revised Bloom Taxonomy [8]. 
The comparison of Bloom Taxonomy tables and its revisions in the cognitive domain is shown below.

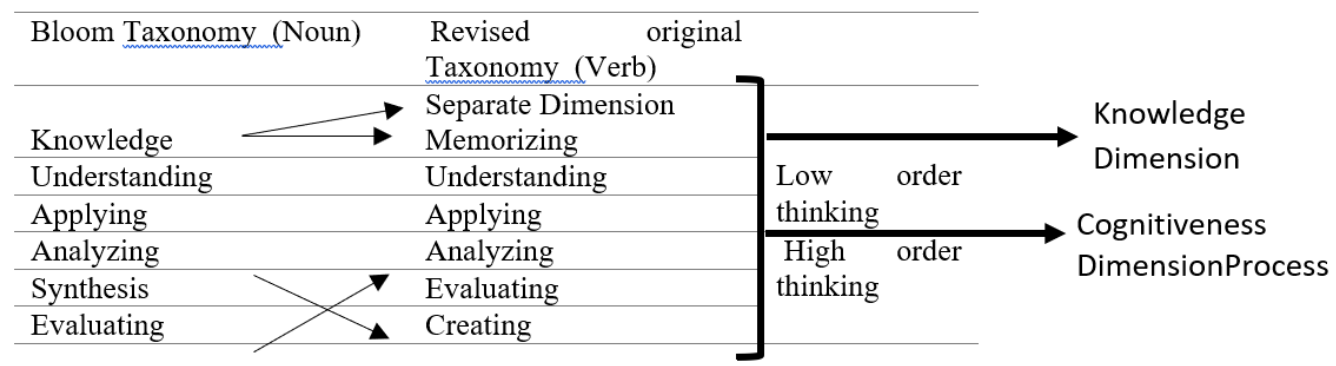

Fig. 2. Series of changes to the Taxonomy Category from original framework to its revision by Andersen and Krathwohl (2010)

\section{Result and Duscussion}

The Focus in examining questions in fashion learning is by using revision to improve critical thinking namely high order thinking at the stage of applying.

\section{C3 (Application)}

At this phase application is interpreted as information application ability to apply the information at the real situation. Students are hoped to be able to apply their concept and principle to a condition that is new and never given before.

\section{Application Questions:}

Develop the basis form of clothing based on your fashion research!

Study: students can answer these questions using the basis fashion knowledge and correlate it with the urgency of problem. Then, students try to explore the knowledge and experience as well as imagination they may have to discover the self-potential. These conditions bring new challenges for them to solve learning problem.

\section{C4 (Analysis)}

In this phase Analysis is considered as an ability to break down a material into clearer components. These abilities could be:

1. Element analysis (analysis of parts of the material)

2. Relationship analysis (relationship identification)

3. Analysis of organizing principles/organizational principles (organizational identification)

In this phase, learners are asked to decipher information into several sections to find assumptions, distinguish opinions and facts and find causal links. Operational verbs that can be used at this level are analyzing, auditing, solving, emphasizing, detecting, diagnosing, selecting, detailing, nominating, charting, correlating, rationalizing, testing, enlightening, exploring, imagining, concluding, finding, analyzing, maximizing, ordering, editing, linking, choosing, measuring, training and transferring. 
Applications questions:

All designs and projects are initiated with identification of needs with research and investigation methods regarding relevant information. It has been related how a fashion product can have a mindset that can meet the criteria of what is called creativity and innovation.

Study: Form of Questions requires students to know not only from identification of problem but also to comprehend the method used by the research method in solving the problem applicative. Finally, students can raise analysis that can be explained descriptively. Departing from this condition, students are expected to be able to practice their cognition by tracing the facts to find connection between the causes and the effect as a result of a relationship.

\section{C5 (Evaluation)}

At this level, evaluation is defined as the ability to assess the benefits of one thing for a particular purpose based on clear criteria. This activity deals with the value of an idea, creation, ways or method. At this stage, someone is guided to gain new knowledge, better understanding, new applications and unique new ways of analysis and synthesis. According to Bloom there are at least 2 types of evaluation, namely: 1. Evaluation based on internal evidence 2. Evaluation based on external evidence. At this level, students evaluate information including making decisions and policy. Operational verbs that can be used in this level are: comparing, concluding, evaluating, directing, criticizing, weighing, deciding, separating, predicting, clarifying, assigning, interpreting, defending, detailing, measuring, summarizing, proving, validating, testing, supporting, choosing, and projecting.

\section{Application Questions:}

Criticize 3 sources of information with a central issue that can inspire you to be a research theme!

Study: Questions in the form of test questions lead students to examine more deeply about the given topic. Students can answer this question if he can elaborate indicators from questions one by one. Next, do the problem formulation and then find a solution to the problem by finding answers. A much deeper understanding of the situation will be formed, or the problems faced in executing and making decisions. Next, students are expected to use this understanding to perform some applications based on the abilities with various efforts in doing the analysis.

\section{C6 (Create)}

At this level, synthesis is interpreted as the ability to produce and combine elements to form a unique structure. This ability can be in the form of producing unique communications, plans or activities that are intact, and a set of abstract relationships. At this level Create emphasizes on cognitive processes, directing students to produce a new product by organizing several elements into a pattern that are different from before. Students need a meaningful learning experience in the learning process they are going through. The activity of creating leads to the process of creative thinking, but that does not mean that students have total ability to create.

At this level, students are required to produce their own hypotheses or theories by combining various sciences and knowledge. Operational verbs that can be used in this level are: abstract, organize, animate, collect, categorize, code, combine, compose, compose, construct, cope, connect, create, create, correct, correct, design, plan, dictate, enhance, clarify, clarify facilitate, form, formulate, generalize, combine, integrate, limit, repair, display, prepare, produce, summarize and reconstruct. 
Application Questions:

Create an interesting theme about creative and innovative ideas. Use brainstorming ideas to find themes with information that is relevant to innovation!

Study: The question item in the form of the question above requires students to determine a design theme that starts with research activities. The initial research used was mapping the problem using mind maps (mind mapping) using 'spider diagrams'. The challenge of this problem is that students must describe the innovations they find. At this stage students are required to master broad insights and will face adaptation to cultural, environmental, and lifestyle issues that can be obtained from information from a variety of print and electronic media. Finally, students can find interesting themes that can be interpreted into designs that have innovation value.

\section{Conclusion}

Application of the revised taxonomy provides benefits for lecturers to cultivate critical thinking. The revised taxonomy categorized learning activities that can be used to achieve learning objectives following assessment to measure abilities mastered by students. Revised Bloom's Taxonomy makes learning goals easier and clearer in its use. Exploration that has been carried out in this revision gives deeper attention to the cognitive knowledge to solve problems.

The readiness of fashion design education is expected to support the creative industries that demand critical thinking to all changes. Implementation of adaptation to the professional environment can be started from classroom learning by implementing exercises that require high-level thinking skills. In addition, the internalization of the revised Bloom Taxonomy into Fashion Design learning can help lecturers to understand the learning objectives, make better decisions about teaching and be able to access students within the goals framework.

The revision table is very helpful to determine the accuracy in adjusting the arrangement between the objectives that have been set so that it is aligned with the assessment used and the desired learning outcomes.

\section{References}

[1] De Felice F and Petrillo A. Key success factors for organizational innovation in the fashion industry International Journal of Engineering Business Management 5 5-27. (2013).

[2] Kanematsu H and Barry D M. STEM and ICT Education in Intelligent Environments: Springer) pp 9-13. (2016).

[3] Krathwohl D R. A revision of Bloom's taxonomy: An overview Theory into practice 41 212-8. (2002).

[4] Lee Y-J, Kim M, Jin Q, Yoon H-G and Matsubara K. East-Asian Primary Science Curricula: Springer) pp 11-6. (2017).

[5] Woolfolk A. Educational Psychology vol Thirteenth Edition (England: Pearson Education). (2016).

[6] Fan J-Y, Feng D-P and Lai M-H. The Application of Idea Generating Approach: A Case Study from Fashion Design. In: International Conference on Social Science and Humanity IPEDR. (2011). 
[7] Karpova E, Marcketti S and Kamm C. Fashion industry professionals' viewpoints on creative traits and, strategies for creativity development Thinking Skills and Creativity 10 159-67. (2013).

[8] Davis P M. Cognition and LearningA Review of the Literature with Reference to Ethnolinguistic Minorities. SIL e-Books (2014). 